\title{
Wireless Wearable Self-calibrated Sensor for Perfusion Assessment of Myocutaneous Tissue
}

\author{
Melissa Berthelot, Ching-Mei Chen, Guang-Zong Yang, and Benny Lo
}

\begin{abstract}
Blood flow and perfusion monitoring are critical appraisal to ensure survival of tissue flap after reconstructive surgery. Many techniques have been developed over the years: from optical to chemical, invasive or not, they all have limitations in their price, risks and adaptiveness to the patient. A wireless wearable self-calibrated device, based on near infrared spectroscopy (NIRS) was developed for blood flow and perfusion monitoring contingent on tissue oxygen saturation $\left(\mathrm{StO}_{2}\right)$. The use of such device is particularly relevant in the case of free flap myocutaneous reconstructive surgery; postoperative monitoring of the flap is crucial for a prompt intervention in case of thrombosis. Although failure rate is low, the rate of additional surgery following anastomosis problem is about 50\%. NIRS has shown promising results for the monitoring of free flap, however lack of adaptation to its environment (ambient light) and users (body mass index (BMI), skin tone, alcohol and smoking habits or physical activity level) hinders the practical use of this technique. To overcome those limitations, a self-calibrated approach is introduced. Tested with ischaemia and cold water experiments on healthy subjects of different skin tones, its ability to personalize its calibration is demonstrated. Furthermore, using a vascular phantom, it is also able to detect pulses, differentiate venous and arterial colouredlike fluids with distinct clusters and detect significant changes in simulated partial venous occlusion. Placed in the trained classifier, partial occlusion data showed similar results between predicted and true classification. Further analysis from partial occlusion data showed that distinct clusters for $\mathbf{7 5 \%}$ and $100 \%$ occlusion emerged.
\end{abstract}

\section{INTRODUCTION}

Blood flow and perfusion monitoring is one of the first act performed by the surgical team following reconstructive surgery after trauma or transplant. In particular, myocutaneous free flap (also called skin graft) operation is a common operation for soft tissue reconstruction in breast, head and neck or lower limb; for example, about 600 reconstructions have been performed for both breast, head and neck free tissue transfer between February 2012 and December 2015 at Charing Cross Imperial Hospital. This operation requires the extraction of all skin layers with blood vessels to be placed where reconstruction is needed: the grafted blood vessels are anastomised. Failure in the anastomosis would result in the death of the skin graft and the patient would need to undergo additional surgery. Flap failure is usually identified in the first 24 to 48 hours following surgery. Close monitoring is therefore essential to prevent failure and increase flap salvage rate. There is no standard method for postoperative free flap monitoring. The assessment of viability of the flap is usually carried out by visually inspecting the flap colour

The Hamlyn Centre, Imperial College London, UK \{meb14,g.z.yang, benny.10\}@imperial.ac.uk and texture, skin turgor or capillary refill time. Assessed at regular interval, such assessment is highly subjective and dependent on the experience of the surgical team. It is often not sufficient to spot early failure and increase flap salvage rate as blood flow disruption can happen at anytime. The postoperative care team cannot continuously assess the flap despise their effort. Different technological approaches have been proposed for continuous blood flow monitoring. Thermal monitoring is one of the first technique used, however, environmental factors significantly affect the accuracy of the measurements. Studies by Khouri \& all [1] showed that such method is not reliable over different situations and patients, as it would require strict regulation of parameters, such as room temperature, patients clothing and movements, and the use of a control temperature source near the flap. Invasive methods based on biochemical sensing, such as microdialysis or tissue oxygen tension, showed promising results, particularly in the case of postoperative buried flaps monitoring or neurosurgery. Although continuous assessment is provided with high sensitivity to change according to the concentrations of the investigated proteins, there is a lack of studies demonstrating their efficiency and reliability over their price, bulkiness and risks to the patients [2], [3]. Implantable Doppler, based on ultrasound is popular for both buried and not-buried free flap: high flap salvage rate are recorded [4]. However, its price and the fact that the probe is usually left in the patient as well as its delicate establishment around the blood vessel might make the use of its non invasive equivalent (laser Doppler flowmetry) more attractive. Laser Doppler can only be used for not buried free flap, in addition to discrete assessment, its use requires practice and experience to interpret the results as it is highly sensitive to movement, with an unknown penetration depth and background noise of the surrounded blood vessels. The near infrared spectroscopy (NIRS) optical technique has derived from pulse oximetry. Focusing on haemoglobin that are the proteins carrying oxygen to the different organs, oxygenation level can be measured [5] directly in the blood vessels or in the skin.

The objective of this paper is to introduce a new wireless wearable device for blood flow and perfusion assessment for free flap autologous reconstruction. In addition to its miniaturised size, light weight and wireless communication, the device is self-calibrated as it adapts itself to acquire signals independently to the user and environmental conditions. To demonstrate the different abilities of the device, experiments on healthy subjects and on vascular phantom have been conducted. 


\section{SELF-CALIBRATED TISSUE OXYGENATION MONITORING SENSOR}

Following our previous work [6], a miniaturised (11 mm X $18 \mathrm{~mm}$ ) wireless optical device based on NIRS has been developed. Based on photoplethysmogram (PPG), it is mainly composed of one red led $(660 \mathrm{~nm})$, one infrared (IR) LED $(940 \mathrm{~nm})$ and a photo-detector as depicted in figure 1. As pointed out by M.W Wukitsch \& all [5], wavelengths around $660 \mathrm{~nm}$ are mainly absorbed by oxygenated haemoglobin $\left(\mathrm{HbO}_{2}\right)$, while those around $940 \mathrm{~nm}$ are absorbed by deoxygenated haemoglobin ( $\mathrm{HHb})$. Consequently, blood flow and perfusion through the skin can be assessed. Oxygenation saturation in the skin $\left(\mathrm{StO}_{2}\right)$ is calculated by $\mathrm{StO}_{2}=$ $\mathrm{HbO2} / \mathrm{THb}$ (1), with $T H b$, the total haemoglobin defined as $\mathrm{THb}=\mathrm{HbO}_{2}+\mathrm{HHb}$. The proposed device overcome the limitations of conventional flap monitoring instruments, as it is non-invasive, wireless, light weighted and can be attached anywhere on the body. Directly connected to a mobile application via Bluetooth Low Energy (BLE) and a cloud server, multiple personnel of the surgical team can remotely access the data and monitor the flap simultaneously. An on-node self calibration mechanism is designed for adapting the sensing for both the user and the surrounding environment. Figure 2 represents the self-calibration control mechanism of the device. The main parameters involved in the calibration process are the gain of the amplifier and the intensities of the LEDs. This self-calibration overcomes many limitations such as ambient light interferences (eliminated by passive cancellation [5]), temperature that influences vascular response, skin tone - mostly based on skin pigmentation [7] and skin thickness. To validate the device's ability in self-calibration, two healthy subject studies were conducted. Ethical approval was granted by the NHS South East London Research Ethics Committee 3 in December 2010 (10/H0808/124). The first is an ischaemia experiment performed on healthy volunteers of different skin tones: the investigated device was placed on the subjects forearm. The Fitzpatrick scale, which is a classification of six types of human skin tone to predict response to ultraviolet (UV) light - it directly provides information on skin pigmentation was used to categorised the skin tone of each participant. Specifically, the Fitzpatrick scale defines a type 1 as a red/blonde haired person with green eyes and very light skin; type 2 as a light to medium haired person with light to medium coloured eyes and light to medium skin; type 3 as a medium coloured haired person with medium to dark eyes and medium to olive skin; type 4 as a dark haired person with dark eyes and dark olive to light brown skin; type 5 as a dark haired person with dark eyes and a dark skin; type 6 as a dark haired person with dark eyes and very dark skin. One person of each category was recruited (6 persons in total). All volunteers were female aged from 20 to 25 years old, with no smoking or alcohol prior, a BMI between 18.5 and 24.9 (healthy) and practising in average 2 hours of sport a week. The protocol consisted in acquiring a baseline signal after calibration, then, using a sphygmomanometer, blood

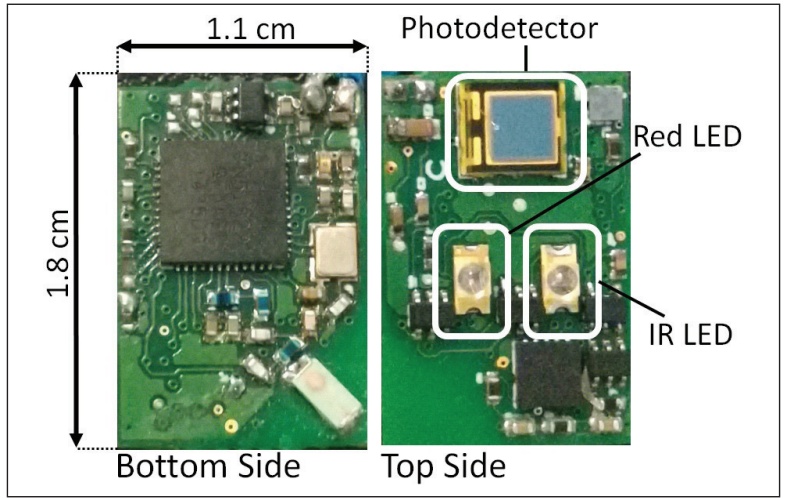

Fig. 1. Bottom ans top views of the device.

flow was disrupted with a pressure set at $70 \mathrm{mmHg}$ followed by pressure release, then, a pressure of $100 \mathrm{mmHg}$ and finally pressure release. The second experiment was performed on the same healthy volunteers. After acquiring a baseline signal after calibration, the participant placed one's hand in cold water, finally data was acquired with one's hand out of the cold water.

Figure 3 shows the results for the ischaemia experiment and figure 4 shows the cold water experiment results for all participants. As expected, temperature and slow blood flow induce a decrease in the $\mathrm{StO}_{2}$ percentage. Over the different type of skin tones, the overall slope of the curves are similar. Specifically, the correlation coefficient is 0.709 for types $1-2$, is 0.583 for types $1-3$, is 0.572 for types $1-4$, is 0.564 for types $1-5$ and is 0.352 for types $1-6$. Similarly, the correlation coefficient is 0.824 for types $2-3,0.819$ for types $2-4,0.448$ for types $2-5$ and 0.250 for types $2-6$. The correlation coefficient is 0.704 for types $3-4$, is 0.388 for types $3-5$ and is 0.293 for types $3-6$. The correlation coefficient is 0.364 for types $4-5$ and 0.161 for types $4-6$. Finally, the correlation coefficient is 0.729 for types 5-6. As expected, the closest the types of skin tone are, the higher the correlation coefficient is. Those experiments demonstrates the adaptivity of the device over different situations and skin tones with similar results.

\section{FLUID FLOW ASSESSMENT ON A VASCULAR PHANTOM}

To further assess the ability of the device to detect and assess blood flow disruption, further experiments using a vascular phantom were conducted. The vascular phantom used was made of silicone skin from Nusil MED-4211 mixed with skin dye; tubes of $2 \mathrm{~mm}$ of inner diameter were introduced inside the silicone to simulate venous and arterial blood vessels [6].

\section{A. Pulse Detection \& Coloured Fluid Distinction}

Pulse detection is crucial in many medical applications. Particularly to free flap surgery, being able to detect pulse is important to ensure that no thrombosis has occurred. For example, implanted and laser Doppler flowmetry provide direct information about pulse and its strength. In the case of reduced signal strength or its complete disappearance, 


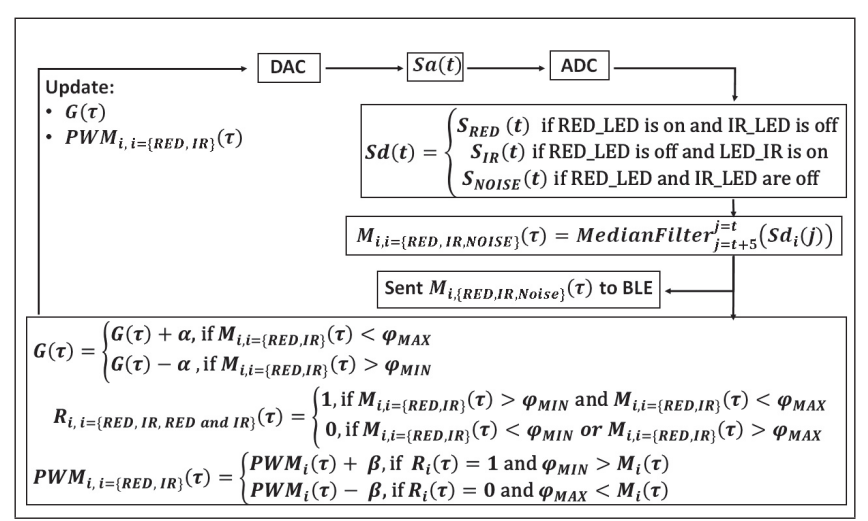

Fig. 2. On node automatic gain control of the device. DAC=digitalanalogue converter; $\mathrm{ADC}=$ analogue-digital converter; $\mathrm{PWMI}, \mathrm{I}=\{\mathrm{RED}$, IR $\}=I$ LED reading; $\mathrm{G}=$ gain of the ampli op; $\mathrm{Sa}=$ analogue output of the amplifier; $S d=$ digitalised $S a$ signal; RI, I $=\{$ RED, IR $\}=$ register saving calibration parameters for $\mathrm{I}$; MI, I $=\{\mathrm{RED}, \mathrm{IR}\}=\mathrm{SdI}$ result from median filtering; BLE=Bluetooth Low Energy; $\alpha=0.87 \mathrm{mV} ; \beta=13 \mathrm{mV} ; \varphi_{\min }=86 \mathrm{mV}$ $; \varphi_{\max }=2 \mathrm{~V}$

anastomosis problem is highly suspected. Using a syringe, at regular intervals, fluid was inserted into a tube to simulate pulses. Figure 5 shows the simulated $\mathrm{StO}_{2}$ level measured during the experiment. At each pulse, referenced by an arrow on figure 5, the simulated $\mathrm{StO}_{2}$ percentage based on equation (1) decreases then increases back close to its baseline level. Those results are coherent with previous results compared to the gold standard $\mathrm{O} 2 \mathrm{C}$ machine [6]. To further evaluate the ability of the device to assess blood flow, two synthetic venous and arterial blood fluids from Bloody Marvellous were used. Because of the reduced amount of oxygen, venous blood is darker than arterial blood. In the case of free flap surgery, thrombosis in artery or venous vessel would result in ischaemia or swelling and cause a change in the color of the flap. This change would eventually be spotted by the postoperative care team during the regular assessments, however not immediately after its occurrence as factors such as temperature, size of the flap and perfusion speed might delay its discovery. Prompt detection of blood vessel thrombosis is critical to enable flap salvage. Different methods already exist in the detection of color difference between arterial and venous flow. Mainly based on optical assessment, such as special CCD camera [8], most of them are bulky and expensive systems that cannot easily be used for a continuous postoperative monitoring. To replicate consequences of thrombosis effect, synthetic arterial and venous blood were introduced into the same tube at regular interval. Figure 6 shows the simulated $\mathrm{StO}_{2}$ percentage based on equation (1) during the experiment. During the introduction of synthetic arterial blood, there is a drop in the percentage values, while it increases when synthetic venous blood is introduced. Based on the results, a naive Bayes classifier was used to categorize the response of the device to arterial and venous flow; figure 7 shows the classification. Whilst the response to synthetic arterial flow is spread, the response to venous-like stimulation is clustered on a specific area.

The overlap of the Gaussian contours might come from the possible inexactitude of the start and end of the introduction

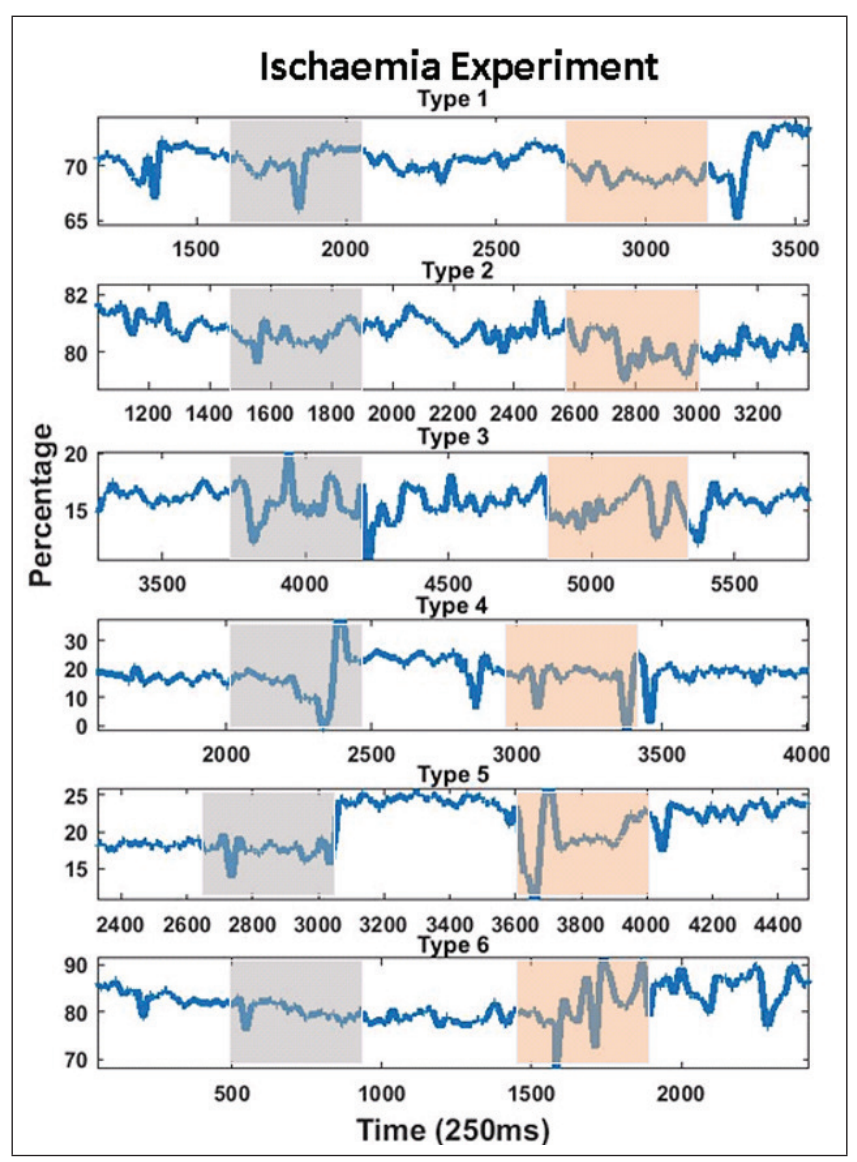

Fig. 3. Ischamia experiment for all types of Fitzpatrick scale. The grey area is $70 \mathrm{mmHg}$ pressure applied, while the one on red is for $100 \mathrm{mmHg}$.

of the synthetic arterial and venous blood into the vascular phantom, from which the training is based. From this model, future data can be classified into the corresponding class for distinction between arterial or venous thrombosis based on light extinction.

\section{B. Partial Occlusion Detection}

As highlighted by Ponticorvo \& all [9], venous occlusion study has been neglected over the years. However, venous occlusion is the most common cause of free flap failure and should be prioritized for monitoring. Indeed, in the case of arterial thrombosis, the venous blood flow will stop in minutes (because there is no more blood to evacuate in the flap), while in the case of venous thrombosis, arterial blood flow will remain constant for a significant amount of time before stopping [10], [4], [9]. Considering that thrombosis is an evolving phenomenon, early detection would significantly improve flap salvage rate. Previous studies lead by Ponticorvo \& all [9] on pigs and Gimbel \& all [11] on rabbits evaluated the feasibility of detecting partial occlusion using spatial frequency domain imaging (SFDI), laser Doppler, NIRS and transcutaneous partial pressure of oxygen $\left(\mathrm{TpO}_{2}\right)$ and carbon dioxide $\left(\mathrm{TpCO}_{2}\right)$. Results show that NIRS and $\mathrm{TpO}_{2}$ cannot distinguish between baseline and partial occlusion [11]. However, the partial occlusion in the latter paper was not well defined accordingly to the baseline and the data were taken discretely (5 points over 25 minutes). Using 


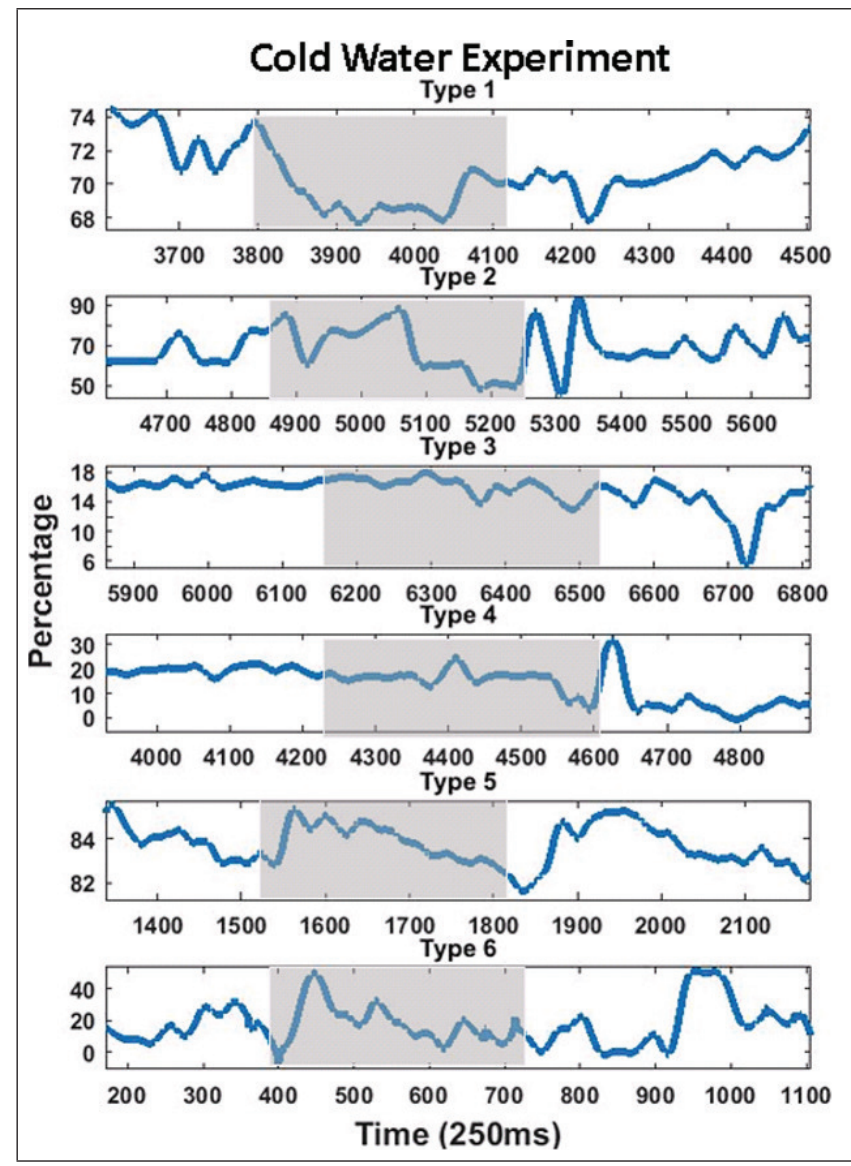

Fig. 4. Cold water experiment for all types of Fitzpatrick scale. The grey area shows the data for which the participant placed her hand in cold water.

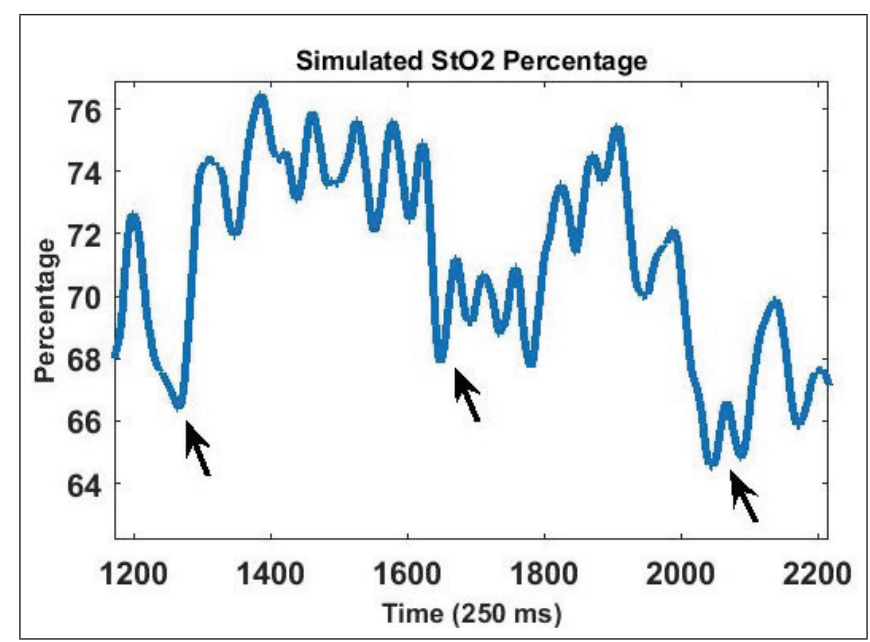

Fig. 5. Simulated $\mathrm{StO}_{2}$ percentage during pulse experiment. At each pulse, referenced by an arrow, there is a drop in the percentage level followed by an increase.

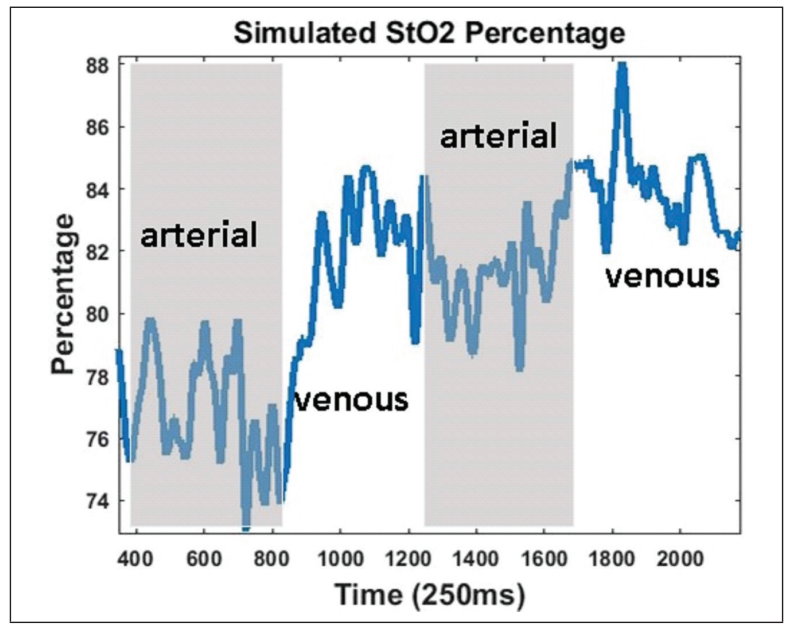

Fig. 6. Simulated $\mathrm{StO}_{2}$ percentage during synthetic arterial and venous blood experiment.

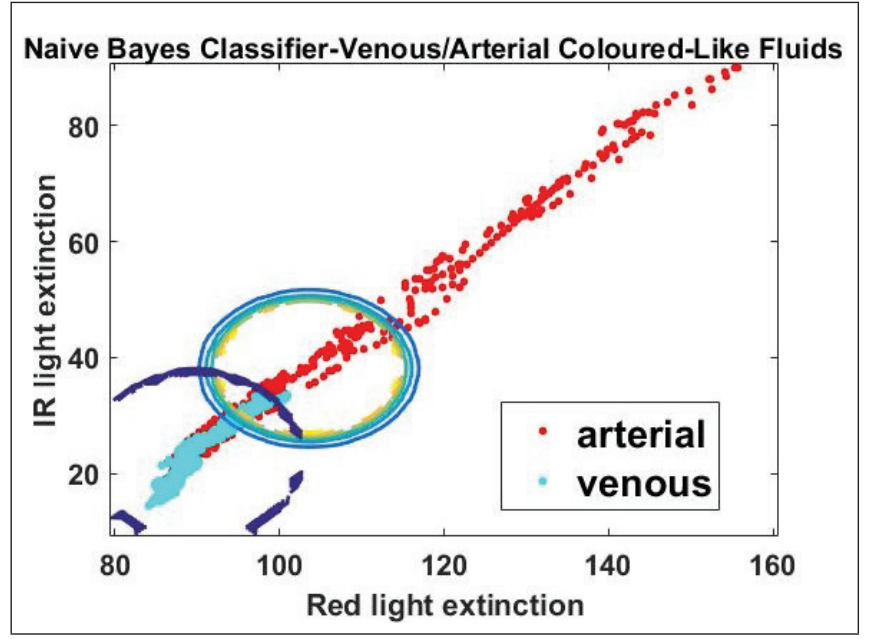

Fig. 7. Naive Bayes classification of the data during the experiment of synthetic venous and arterial blood distinction (see figure 6).

the pump Ismatec Reglo ICC, synthetic arterial and venous flows were simulated: the baseline was set in such a way that synthetic arterial blood was pumped at $6.8 \mathrm{ml} / \mathrm{min}$ going in the right direction, while synthetic venous blood was pumped at $4.5 \mathrm{ml} / \mathrm{min}$ going in the left direction. Partial occlusion of the venous blood flow was simulated at $25 \%, 50 \%, 75 \%$ and $100 \%$, resulting in $3.4 \mathrm{ml} / \mathrm{min}, 2.3 \mathrm{ml} / \mathrm{min}, 1.1 \mathrm{ml} / \mathrm{min}$ and $0 \mathrm{ml} / \mathrm{min}$ of blood flow respectively. Although baseline for arterial and venous flow was found in the literature [9], no literature on quantitative arterial blood flow measurements have been found during partial venous occlusion or vice versa, therefore, the arterial blood flow was kept constant during the experiment. Figure 8 shows the simulated $\mathrm{StO}_{2}$ based on equation (1) during the experiment; at regular interval, starting with baseline, $25 \%$ occlusion, release, $50 \%$ occlusion, release, $75 \%$ occlusion, release, $100 \%$ occlusion blood flows were simulated. The simulated release correspond to a $0 \%$ occlusion or a baseline flow. As expected, the simulated $\mathrm{StO}_{2}$ decreases as the partial occlusion increases. Comparing the results from the different occlusions with the baseline using a paired t-test at $\mathrm{p}$-value under $1 \%$, the device is able to significantly differentiate all occlusion percentages 


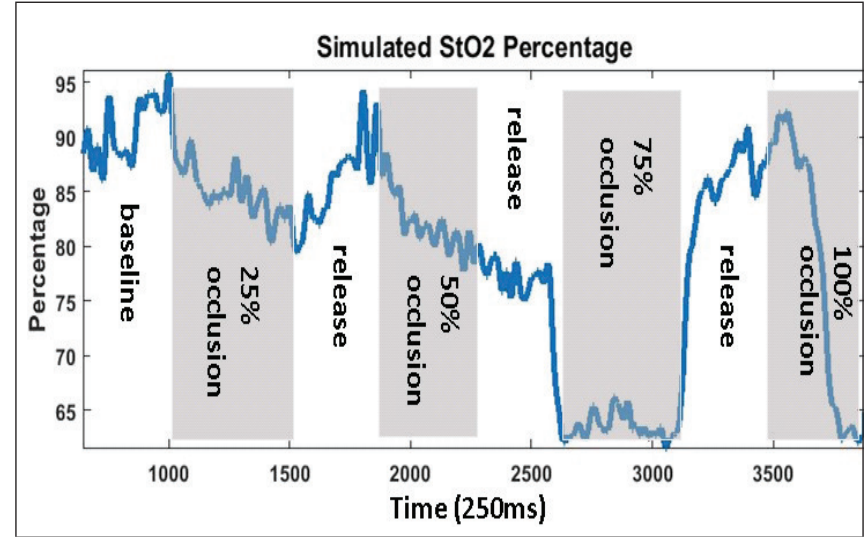

Fig. 8. Simulated $\mathrm{StO}_{2}$ percentage during synthetic venous flow occlusion experiment. From a baseline, impact of partial venous occlusion (for $25 \%$, $50 \%, 75 \%$ and $100 \%$ ) is monitored. Increasing the occlusion percentage, decreases the simulated $\mathrm{StO}_{2}$ percentage values.

for the simulated $\mathrm{St}_{2}$ percentage based on equation (1), red light extinction, IR light extinction and the sum of red and IR lights extinctions parameters, which respectively simulate $\mathrm{HbO}_{2}, \mathrm{HHb}$ and $\mathrm{THb}$ concentrations. As occlusion affects the amount of $\mathrm{HbO}_{2}$ and $\mathrm{HHb}$, and consequently the overall color of the flap, the data were placed into the previously trained Bayes classifier (see figure 7). Figure 9 shows the predicted and true classifications for the partial occlusion data. In addition to accumulated errors in the trained data and in the true classification labelling due to inexactitude in the start and end of the synthetic blood and partial occlusion introductions, while the arterial classes of the predicted and true classifications have a similar scattering, the true venous class is more scattered than predicted. The true venous counts $41 \%$ of observations while the prediction holds $67 \%$ of them. Within $53 \%$ of similar results between the true and predicted classifications, there is $59 \%$ of same venous instances between the two classifications. For $25 \%$ and $100 \%$ venous occlusion, there is respectively $31 \%$ and $100 \%$ of similarities between the true and predicted classification. Further classification of the level of venous occlusion using a naive Bayse classifier showed that release, $25 \%$ and $50 \%$ venous occlusion classes are clustered together while $75 \%$ and $100 \%$ clusters are highly distinguishable from it (see figure 10). Although it is possible to distinguish $75 \%$ and $100 \%$ venous occlusions, the contours of the Gaussian are overlapping (possibly due to inexactitude in the start and end of the data classes used in the classification labelling). In addition to the distinct clusters of venous and arterial synthetic blood (see figure 7), within the venous cluster, partial occlusion can be detected with significant simulated $\mathrm{St}_{2}$ percentage for every venous partial occlusion and from $75 \%$ of venous partial occlusion classification (see figure 10). Those results are promising for further distinction of arterial or venous partial occlusions based on optical sensing and the analysis of $\mathrm{StO}_{2}$ percentage and arterial/venous and level of occlusion classification.

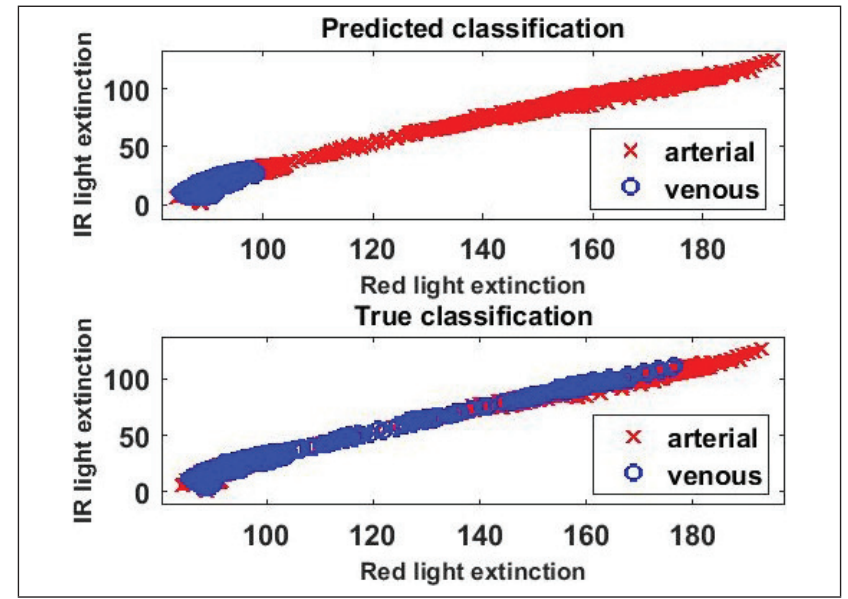

Fig. 9. True and predicted classifications for the partial occlusion experiment data into the previously trained naive Bayes classifier (see figure 7)

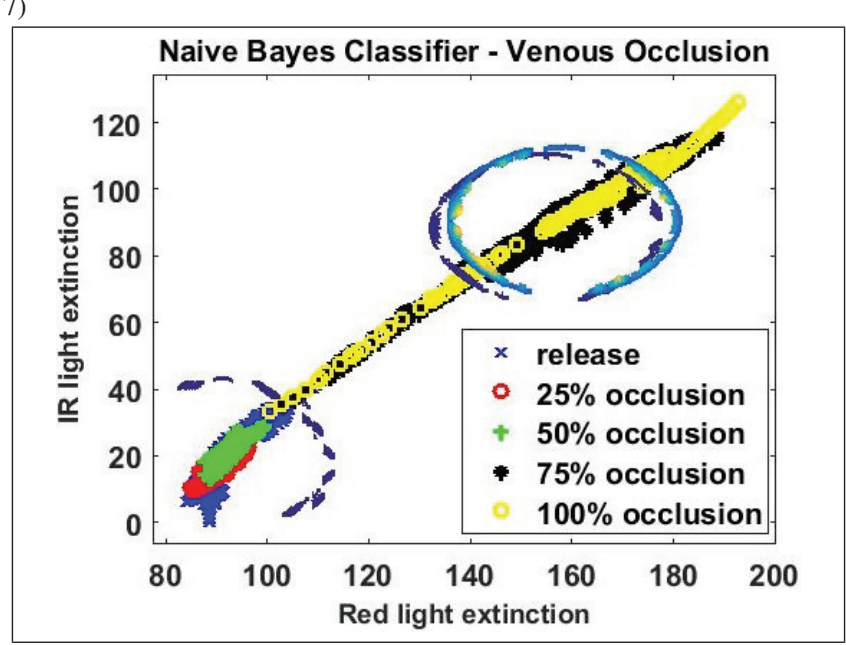

Fig. 10. Naive Bayes classification for the partial synthetic venous occlusion experiment data.

\section{DISCUSSION}

Although failure rate of free flap reconstructive surgery is low, close monitoring is crucial for prompt intervention in case of anatomosis problem. Currently, no standard method among hospitals exists for monitoring the flap's condition; based on visual observations and experience, intermittent assessments of the flap are not sufficient to prevent flap failure. Constant monitoring would reduce flap failure rate and additional surgery by providing real-time information on flap viability. The existing devices and methods for continuous monitoring of the flap viability have limitations in their price, risks and ability to adapt to the patient and one's close environment [8], [2], [3]. A wearable wireless device with self-calibration for $\mathrm{StO}_{2}$ monitoring was introduced. Small, light weighted with possibility to attach it on any location of the body, it aims to overcome limitations of the existing devices. With a mobile application and cloud server, members of the postoperative care team can remotely monitor the condition of the flap, which enables early intervention in case of potential failure.

To demonstrate the device's ability to detect changes in blood perfusion in tissue, different experimentations were 
performed. A first experiment showing adaptiveness of the device over different skin tones based on Fitzpatrick scale was conducted. Results are consistent to previous work [6] for which results were compared with a gold standard $\mathrm{O} 2 \mathrm{C}$ machine. A second experience showed the ability of the device to adapt to impacts of temperature on the vascular system. Despite the non-absolute values of $\mathrm{StO}_{2}$ percentage, those standard experiments conducted on volunteers with different skin tones provide key references for relative measurements on oxygenation level. Further work will be required to derive the transformation of the sensor reading into absolute $\mathrm{StO}_{2}$ measurements.

Using a vascular phantom with synthetic arterial and venous blood, the device was able to 1) detect pulses, 2) differentiate synthetic arterial from venous flow with distinguishable clustering using naive Bayse classifier and 3) significantly distinguish baseline blood flow from different synthetic venous partial occlusion - from $25 \%$ to $100 \%$ occlusion compared to the baseline - with highly distinguishable clusters between release and low percentage venous occlusions (25\% and $50 \%$ ) and release and high percentage venous occlusions (75\% and 100\%). Pulse detection is an important feature for blood flow monitoring, particularly to confirm anastomosis viability. Similarly, in the case of arterial or venous thrombosis, the general color of the blood in the flap and later the color of the flap itself will defer accordingly. Differentiating venous from arterial blood is therefore important to ensure prompt intervention in case of thrombosis. Results showed that the device is able to differentiate synthetic arterial blood from venous blood with higher simulated $\mathrm{StO}_{2}$ percentage in the case of synthetic venous flow. Further analysis showed that those two classes are distinguishable using a naive Bayse classifier. To extend the simulation closer to reality, partial venous occlusion was emulated to determine the ability of the device to detect it. Increasing venous partial occlusion from $25 \%$ to $100 \%$, at $25 \%$ step, significant detection of all partial occlusion was found. Using this dataset to be classified on the previous Bayse classifier, results show similar classification among the true and predicted labellings. Further analysis on the partial occlusion data showed that from the five classes (release, 25\%, 50\%, 75\% and 100\% occlusions), three main clusters are arisen composed of 1) release, $25 \%$ and $50 \%$ occlusion, 2) $75 \%$ occlusion and 3) $100 \%$ occlusion. Merging those results and focusing on venous cluster, distinction of partial venous occlusions can therefore be performed.

Although the results were obtained from phantom simulation, they show promising characteristics for blood flow and perfusion monitoring using the proposed optical sensor. Extended research on animals needs to be carried out to ensure the reliability of the found classification model. Further work on power consumption for longer battery life is also crucial to optimize pervasiveness of the device for the patients during the 24 to 48 hours of postoperative monitoring. Finally, miniaturization of the device is intended with possible addition of LEDs for detection of other blood flow parameters. Translation of this platform into implantable device to directly be placed around venous blood vessel would also allow postoperative monitoring of any type of flap.

\section{CONCLUSIONS}

A new wireless wearable device was introduced: light weighted, small, pervasive and with ubiquitous access to data, its ability to provide continuous assessment of blood flow and perfusion states has been demonstrated. Selfcalibrated, the device adapts itself to the user and one's surrounding environment. Further assessments on animals and patients who underwent not buried free flap surgery are planned.

\section{ACKNOWLEDGMENT}

This work is supported by the UK National Institute for Health Research (NIHR) Imperial Biomedical Research Centre (BRC) - A reflective photoplethysmographic plaster for monitoring soft tissue viability after free autologous tissue flap transfer. The authors would like to thank Mr. Shehan Hettiaratchy of the Imperial College Healthcare NHS Trust and Dr. Daniel Leff of the Department of Surgery and Cancer, Imperial College for their support and advices on the studies.

\section{REFERENCES}

[1] R. K. Khouri and W. W. Shaw, "Monitoring of free flaps with surfacetemperature recordings: Is it reliable?.," Plastic and reconstructive surgery, vol. 89, no. 3, pp. 495-499, 1992.

[2] C. Mourouzis, R. Anand, J. R. Bowden, and P. A. Brennan, "Microdialysis: Use in the assessment of a buried bone-only fibular free flap," Plastic and reconstructive surgery, vol. 120, no. 5, pp. 1363-1366, 2007.

[3] N. Sinis, H. Rennekampff, M. Haerle, and H.-E. Schaller, "Free flap monitoring with continuous tissue oxygen tension measurement," European Journal of Plastic Surgery, vol. 28, no. 8, pp. 507-512, 2006.

[4] T. Poder and P.-H. Fortier, "Implantable doppler in monitoring free flaps: a cost-effectiveness analysis based on a systematic review of the literature," European annals of otorhinolaryngology, head and neck diseases, vol. 130, no. 2, pp. 79-85, 2013.

[5] M. W. Wukitsch, M. M. T. Petterson, D. R. Tobler, and J. A. Pologe, "Pulse oximetry: analysis of theory, technology, and practice," Journal of Clinical Monitoring, vol. 4, no. 4, pp. 290-301, 1988.

[6] C.-M. Chen, R. Kwasnicki, B. Lo, and G.-Z. Yang, "Wearable tissue oxygenation monitoring sensor and a forearm vascular phantom design for data validation," in Wearable and Implantable Body Sensor Networks (BSN), 2014 11th International Conference on, pp. 64-68, IEEE, 2014.

[7] E. Wassenaar and J. Van den Brand, "Reliability of near-infrared spectroscopy in people with dark skin pigmentation," Journal of clinical monitoring and computing, vol. 19, no. 3, pp. 195-199, 2005.

[8] A. Kienle, L. Lilge, I. A. Vitkin, M. S. Patterson, B. C. Wilson, R. Hibst, and R. Steiner, "Why do veins appear blue? a new look at an old question," Applied Optics, vol. 35, no. 7, pp. 1151-1160, 1996.

[9] A. Ponticorvo, E. Taydas, A. Mazhar, T. Scholz, H.-S. Kim, J. Rimler, G. R. Evans, D. J. Cuccia, and A. J. Durkin, "Quantitative assessment of partial vascular occlusions in a swine pedicle flap model using spatial frequency domain imaging," Biomedical optics express, vol. 4, no. 2, pp. 298-306, 2013.

[10] S. Hovius, L. Van Adrichem, H. Mulder, R. Van Strik, and J. Van Der Meulen, "The predictive value of the laser doppler flowmeter for postoperative microvascular monitoring.," Annals of plastic surgery, vol. 31, no. 4, pp. 307-312, 1993.

[11] M. L. Gimbel, M. D. Rollins, E. Fukaya, and H. W. Hopf, "Monitoring partial and full venous outflow compromise in a rabbit skin flap model," Plastic and reconstructive surgery, vol. 124, no. 3, pp. 796803, 2009. 